\title{
Accentuated Eccentric Loading is Superior to Traditional Loading for Improving Acute Countermovement Jump Performance in Adult, Resistance-Trained Males
}

\begin{abstract}
Jared Patus
Clinical Scenario: Traditional loading (TL) is a common technique to employ when engaging in countermovement jumps (CMJ). Accentuated eccentric loading (AEL) is a newer modality that is being explored for acute CMJ performance. Focused Clinical Question: In adult, resistance-trained males, will AEL have a superior impact on acute CMJ performance compared to TL? Summary of Key Findings: The literature was searched for studies that examined the influence of AEL on acute CMJ performance compared to a TL protocol. TL was defined as any loading condition that utilized an equivalent resistance during both the eccentric and concentric contractions. Three studies met the inclusion and exclusion criteria, and were identified and included in the critically appraised topic. Each of the 3 studies found that various AEL conditions were either equal to or better than TL when examining subsequent CMJ performance. In no specific CMJ outcome measure was TL deemed to have a greater impact than AEL. Clinical Bottom Line: AEL provides more favorable acute CMJ performance than TL in adult, resistancetrained males. Strength of Recommendation: Consistent findings from 2 randomized crossover studies and one repeatedmeasured design investigation suggest level $2 \mathrm{~b}$ evidence to support AEL as an ideal protocol for acute CMJ performance.
\end{abstract}

Keywords: athletic performance, training, muscular contractions

\section{Clinical Scenario}

Eccentric resistance training has been shown to induce muscletendon unit (MTU) morphological and architectural adaptations as well as positive improvements in the mechanical function of the trained muscle. ${ }^{1}$ Traditional loading (TL), in which the load is equal during both the eccentric and concentric contractions of a movement, is a common training stimulus. ${ }^{2}$ Recently, more focused attention has been given to the use of accentuated eccentric loading (AEL) and its impact on athletic performance. AEL is the application of an eccentric load that is greater than the prescribed concentric load while employing both the eccentric and concentric contractions and attempting to maintain natural movement patterns. ${ }^{2}$ An example of AEL would be performing the eccentric or downward phase of a squat with an additional external load such as dumbbells and then releasing them to complete the concentric phase.

There are various loading considerations for AEL which range from submaximal AEL conditions, an eccentric to concentric ratio of $70 \% / 50 \%$, to supramaximal loading, an eccentric load greater than the concentric one-repetition maximum. Each of these loading considerations has the potential to elicit various results depending on individual factors such as age, training experience, strength level, or physiological traits. ${ }^{2}$

The use of AEL during explosive movements has been demonstrated to enhance the rate of eccentric force production, coupled with the stimulation of the stretch-shortening cycle to magnify the successive concentric power output. ${ }^{3,4}$ Several studies ${ }^{5-7}$ have provided evidence to indicate that applying AEL loading can enhance the postactivation potentiation (PAP)

The authors (jaredpatus@hotmail.com) is with Concordia University of Chicago, River Forest, IL. response, which is believed to induce subsequent improvements in countermovement jump (CMJ) performance.

\section{Focused Clinical Question}

In adult, resistance-trained males, will AEL have a superior impact on acute CMJ performance compared with TL?

\section{Summary of Search, "Best Evidence" Appraised, and Key Findings}

- The literature search was based on the Oxford Center for Evidence-Based Medicine. ${ }^{8}$

- The literature was searched for studies that examined the influence of AEL on acute CMJ performance compared with a TL protocol. TL was defined as any loading condition that utilized an equivalent resistance during both the eccentric and concentric contractions.

- Three studies ${ }^{5-7}$ met the inclusion and exclusion criteria, and were identified and included in the critically appraised topic (CAT).

- Each of the 3 studies found that various AEL conditions were either equal to or better than TL when examining subsequent CMJ performance. In no specific CMJ outcome measure was TL deemed to have a greater impact than AEL.

\section{Clinical Bottom Line}

Current evidence suggests that the use of AEL for adult (age 19$35 \mathrm{y}$ ), resistance-trained males enhances acute CMJ performance compared with TL. ${ }^{5-7}$ Based on the studies examined, the acute 
time frame following the preloading protocol was deemed to be between 2 and 12 minutes. These studies demonstrated that the optimal time for CMJ performance was between 2 and 6 minutes following AEL conditions. Determining the optimal AEL resistance was not clearly identified from these studies. Using loads of $105 \%$ of one-repetition maximum, $125 \%$ of one-repetition maximum, body mass (BM) plus $5 \%$, and BM plus $20 \%$ were all determined to be more effective than TL. ${ }^{5-7}$ While loads of BM plus $10 \%$ and BM plus $30 \%$ were not significantly better than TL. ${ }^{5-7}$ The evidence generated from the included studies ${ }^{5-7}$ suggests that AEL is more effective at improving acute CMJ performance in adult, resistancetrained males compared with TL protocols.

\section{Strength of Recommendation}

To date, studies relating to this topic are moderate in number, considerable in quality (2b), and fairly consistent in their discoveries. While the studies reviewed in this CAT demonstrated that most of the AEL protocols led to beneficial PAP results for CMJ performance, not every AEL preload weight led to significant improvements. Each of the studies included examined a small number of resistance-trained, adult males (ranging from $n=11-14$ ) in the test design which provides some hesitation for a definite conclusion. However, 2 of the 3 studies employed a crossover randomized design in an attempt to negate potential learning effects. It is for these reasons that the strength of recommendation is scored as B based on the Oxford Center for Evidence-Based Medicine grades of recommendation. ${ }^{8}$

\section{Search Strategy}

\section{Terms Used to Guide Search Strategy}

- Patient/Client group: adult, resistance-trained males

- Intervention (or assessment): AEL

- Comparison: TL

- Outcome(s): acute CMJ performance

\section{Search Terms Used}

Searches included key terms "eccentric loading," "eccentric training," "accentuated eccentric loading," "accentuated eccentric training," "accentuated eccentric loading and jump performance," and "accentuated eccentric loading and countermovement jump performance".

\section{Sources of Evidence Searched (Databases)}

- Google Scholar

- ScienceDirect

- SPORTDiscus

- EBSCOhost

- PubMed

\section{Inclusion and Exclusion Criteria}

\section{Inclusion Criteria}

- Studies from peer-reviewed journals with full text

- Studies published in the last 5 years (2016-2020)
- Studies written in the English language

- Studies that included participants that were healthy and free from a current or recent injury

- Studies that compared preloading jump protocols of AEL to TL

- Studies that measured some form of CMJ performance (power, height, or peak power)

\section{Exclusion Criteria}

- Studies that utilized weeks of AEL training sessions before measuring jump performance

- Studies that measured horizontal jump performance

- Studies focused on females or untrained individuals

- Studies in the form of a meta-analysis or systematic review

\section{Results of Search}

The literature search yielded 105 different articles. From this search, 3 articles were determined to be appropriate for this CAT based on the inclusion and exclusion criteria mentioned in the previous section. The search process is outlined below in Table 1 .

\section{Best Evidence}

The studies summarized in Table 2 provide the best evidence available based on the inclusion and exclusion criteria shown above. All 3 articles were determined to be level $2 b$ evidence based on the criteria from the Oxford Center for Evidence-Based Medicine levels of evidence. ${ }^{8}$ Due to the nature of the intervention, subjects and assessors were not blinded, which in part accounts for the included studies receiving a 6/10 on the Physiotherapy Evidence Database scale.

\section{Summary of Best Evidence}

\section{Implications for Practice, Education, and Future Research}

The objective of this critical appraisal was to examine current research regarding the difference between AEL and TL on acute CMJ performance among adult, resistance-trained males. Two studies examined in this CAT demonstrated that all of the AEL conditions employed in their investigation led to superior CMJ performance when compared with TL. 5,7 The third study found that 1 of the 3 AEL protocols surpassed TL, while the other 2 AEL

Table 1 Summary of Study Designs of Articles Retrieved

\begin{tabular}{|c|c|c|c|}
\hline $\begin{array}{l}\text { Level of } \\
\text { evidence }\end{array}$ & Study design & $\begin{array}{l}\text { Number } \\
\text { located }\end{array}$ & Study \\
\hline $2 b$ & $\begin{array}{l}\text { Crossover randomized } \\
\text { study }\end{array}$ & 2 & $\begin{array}{l}\text { Ong et } \mathrm{al}^{5} \\
\text { Hughes et } \mathrm{al}^{7}\end{array}$ \\
\hline $2 b$ & $\begin{array}{l}\text { Repeated-measures } \\
\text { design }\end{array}$ & 1 & Bridgeman et $\mathrm{al}^{6}$ \\
\hline
\end{tabular}




\begin{tabular}{|c|c|}
\hline Authors & Ong et $\mathrm{al}^{5}$ \\
\hline Study design & Crossover randomized study \\
\hline Participants & $\begin{array}{l}\mathrm{N}=14 \text {, men aged } 19-35 \text { y began and } \\
\text { completed this study. Subjects were active } \\
\text { with at least } 12 \text { mo of lower-body } \\
\text { resistance training. They were determined } \\
\text { to be physically healthy and free from any } \\
\text { current or recent injuries. }\end{array}$ \\
\hline
\end{tabular}
current or recent injuries.

Participants 1RM for the hip sled exercise was measured as a baseline.

Every testing session consisted of a control (ORM) with 0 eccentric loading and eccentric loads at $105 \%$ of $1 \mathrm{RM}$ $(105 \mathrm{RM})$ and $125 \%$ of $1 \mathrm{RM}(125 \mathrm{RM})$ for the hip sled exercise.

A CMJ trial was used to determine the preeccentric value. Next, the posteccentric load of 105RM and 125RM was employed with 6 repetitions for 105RM and 5 repetitions for 125RM.

After eccentric loading occurred, CMJ performance was measured at 3-min intervals over $12 \mathrm{~min}$.

Outcome $\quad 1 R M$ on a hip sled machine was used to measure(s) determine 105RM and 125RM, which were necessary for eccentric loading. CMJ performance was measured by a force plate (Fitness Technology, Adelaide, Australia) to determine $\mathrm{P}_{\text {peak }}$ and a linear position transducer (Fitness Technology) was used to calculate $\mathrm{D}_{\max }$.

Main findings

Level of

evidence

Validity score: $\quad 6 / 10$

No markedly different outcome $(P<.05)$ between ORM and loading conditions of $105 \mathrm{RM}$ and 125RM at baseline. In addition, there was no notable difference between 105RM and 125RM for both $\mathrm{P}_{\text {peak }}$ and $\mathrm{D}_{\text {max }}$ during the interval checkpoints.

At the 3- and 6-min intervals, both 105RM and $125 \mathrm{RM}$ were found to significantly improve $\mathrm{P}_{\text {peak }}$ compared with 0RM. There was a significant reduction in $\mathrm{P}_{\text {peak }}$ between the control of 0RM compared with each of the posteccentric load interval times of 3, 6, 9, and $12 \mathrm{~min}$. At the 3-min interval, $\mathrm{D}_{\max }$ was discovered to be notably higher for both 105RM and $125 \mathrm{RM}$ in comparison with 0RM. PEDro scale

Conclusion

\section{Bridgeman et al $^{\mathbf{6}}$}

Repeated-measures design

$\mathrm{N}=12$, males between the ages of 21 and 29 y started and finished the intervention Participants had at least $2 \mathrm{y}$ of strength training experience, specifically with plyometric training, they could each squat 1.5 times their BW.

Subjects engaged in 2 testing sessions to measure their baseline $\mathrm{CMJ}$ performance and optimum drop height.

DJ and CMJ performance were measured during and after the following 4 protocols: (1) DJ with BM only, (2) DJ with an AEL of $10 \%$ BM, (3) DJ with an AEL of $20 \%$ $\mathrm{BM}$, and (4) DJ with an AEL of 30\% BM. The loads used during AEL DJs were determined as a percentage of each individual's BM.

Each protocol utilized 5 DJ's with the assigned loading resistance followed by a $3 \mathrm{CMJ}$ test at 2-, 6-, and 12-min intervals.

3 maximal CMJs were measured, separated by a 15 -s rest between jumps. Next, the ideal jump height for each individual was determined by employing a variety of drop heights $(28,38,52,62$, and $70 \mathrm{~cm}$ ).

Each CMJ test measured jump height (in centimeters), $\mathrm{P}_{\text {peak }}$ and (GRF[N]) while jump height, contact time (in seconds), flight time (in seconds), and GRF were measured during DJs. Each of the variables was measured with a force place (400S; Fitness Technology).

BM loading and $20 \%$ AEL were found to be superior to $10 \%$ and $30 \%$ AEL for both DJ jump height and DJ flight time.

20\% AEL elicited a significantly greater CMJ height compared with BM, $10 \%$, and $30 \%$ AEL.

CMJ height was determined to be the greatest at 2- and 6-min post-DJs compared with 12-min post-DJ.

$\mathrm{P}_{\text {peak }}$ was significantly greater in the CMJs after the execution of the $20 \%$ AEL.
Hughes et al $^{7}$

Crossover randomized study

$\mathrm{N}=11$, male university students between the ages of 19 and 23 y completed the investigation. Inclusion in the study required participants to have a minimum of $6 \mathrm{mo}$ of resistance training experience, competent $\mathrm{BSq}$ technique (assessed by a certified specialist), and no past of lower limb injury. In addition, a BSq of greater than $150 \%$ of $\mathrm{BW}$ and efficient landing mechanics were assessed.

The first session was used to familiarize participants with the procedures as well as establish baseline testing.

The testing sessions began with a warmup conducive to $\mathrm{BSq}$ and jump squat performance as well as $3 \mathrm{CMJ}$ 's to establish a baseline.

The 2 different preconditioning exercises used were BSq of 3RM and 6 AEL drops ( 3 from the left and 3 from the right) were performed off of a $60-\mathrm{cm}$ box with a load of $5 \% \mathrm{BM}$.

Following the preconditioning loading protocols, a 6-min rest was observed before the execution of 3 more CMJs.

BSq 3RM was determined for each individual before the testing sessions. This value was used for the BSq protocol. $\mathrm{CMJ}$ height (in centimeters) was measured before each loading protocol with 3 CMJs and again 6 min after with 3 more CMJs. All CMJs were done on a mobile contact mat (SMARTJUMP; Fusion Sport, Sumner, Australia).

AEL conditions were found to significantly $(P=.02)$ impact CMJ height compared with BSq conditions.

Post hoc tests demonstrated that AEL had a statistically significant $(P=.03)$ potentiating effect on $\mathrm{CMJ}$ height, whereas the BSq procedure had no significant difference.

The effect size for the increase in $\mathrm{CMJ}$ as a result of AEL was small $(E S=0.31)$ and trivial for $\mathrm{BSq}(\mathrm{ES}=-0.24)$
$2 b$

$6 / 10$

One set of 5 DJs under AEL conditions of $20 \%$ BM produced superior subsequent CMJ performance. Furthermore, 2 min of recovery following the DJs led to the most favorable CMJ outcomes. was found to elicit a significant

improvement in $\mathrm{P}_{\text {peak }}$ at 3 and 6 min after loading compared with ORM. In addition, $105 \mathrm{RM}$ significantly enhanced $\mathrm{D}_{\max }$ at 3 and 6 min with 125RM improving at the 3min interval only.
$2 b$

$6 / 10$

Jump height was found to significantly increase as a result of the AEL protocol, while a BSq protocol did not induce a significant change. Thus, the authors suggested that AEL may be superior to $\mathrm{BSq}$ as a potentiating stimulus for vertical jump performance.

Abbreviations: AEL, accentuated eccentric loading; BM, body mass; BSq, back squat; BW, body weight; CMJ, countermovement jump; $\mathrm{D}_{\text {max }}$, vertical displacement; DJ, drop jump; ES, effect size; GRF, ground reaction force; PEDro, Physiotherapy Evidence Database scale; $P_{\text {peak }}$, peak power; 1RM, one-repetition maximum. 
conditions demonstrated similar results as TL. ${ }^{6}$ Each of the studies was rated as level $2 \mathrm{~b}$ evidence based on the Center of EvidenceBased Medicine's level of evidence. ${ }^{8}$ The outcome from this CAT suggests that adult, resistance-trained males who engage in some form of AEL loading before CMJ, may experience greater jumping performance.

A review of current research about AEL for training and performance concluded that only a small amount of data exists in this field. ${ }^{2}$ However, the authors of this review stated that both acute and chronic responses to AEL have been superior at enhancing strength and power performance. ${ }^{3-7} \mathrm{~A}$ similar study to those examined for this critical appraisal revealed that $\mathrm{CMJ}$ power output, net impulse, and jump height were all significantly influenced by AEL of $30 \%$ BM. $^{9}$ A loading condition of AEL at $20 \%$ of BM did not differ significantly from TL. This study's population was in line with the CAT studies as adult, resistance-trained males were used as subjects.

There are several possible explanations for the improved contractile process following AEL. The first 3, as reported by Walshe et al, ${ }^{10}$ are increased neural stimulation due to a larger stretch of intrafusal muscle fibers from a larger eccentric load, increased stretch of the MTU resulting in greater elastic recoil, and less myofibrillar displacement from reserved elastic energy in muscle fibers at the onset of a concentric contraction. ${ }^{10}$ An additional mechanism that has been proposed is that the intensification of AEL preloading permits the agonist muscles to actively prepare as a part of the cross-bridge is connected before concentric contraction leading to greater force and power production. ${ }^{11}$

The practical implications of AEL's capacity to improve CMJ performance come with some important considerations. First of all, the use of eccentric-based exercise has been linked to the treatment of sports injuries, more specifically tendinopathies. ${ }^{12}$ This review discovered that Achilles and patellar tendon injuries were positively influenced by eccentric exercise. In addition, eccentric exercise has shown promise in the management of other sportinduced tendinopathies. ${ }^{12}$ LaStayo et $\mathrm{al}^{13}$ stated that concentric and isometric contractions have traditionally been the focus of rehabilitation research with eccentric contractions receiving less attention due to their negative association with delayed onset muscle soreness. A recent shift toward the increased use of eccentric contractions in rehabilitation settings has yielded results that are safe and practical-increased muscle size, muscle strength, and physical function. ${ }^{13}$ Thus, the use of AEL in the context of explosive jumping movements may have a connection to Achilles and patellar tendon injuries as they contribute to the jumping motion.

A second practical implication is that preloading with AEL for CMJ performance improves outcomes; however, it does so for only a short time frame. Time frames of 3 to 6,2 , and 6 minutes were deemed to be the optimal postloading times. ${ }^{5-7}$ These limited time frames restrict prospective athletes able to utilize AEL as a means to improve jumping performance. Sports activities with the greatest potential benefit include single-action movements (long jump, high jump, throwing, and diving) and maneuvers where increased peak power and explosive force are required (short sprints, speed skating, and bobsled). Therefore, including AEL in the warm-up for short lived, explosive activities are recommended.

Achieving optimal PAP benefits as a result of an AEL warmup comes with some obstacles. The use of precompetition potentiation effects must be implemented carefully, as not to induce fatigue during a warm-up. Failing to consider the demands of the specific sport (muscle groups involved, type of muscle action, and rest periods) preloading has the potential to adversely impact performance. $^{5}$ The implementation of AEL during an athlete's warm-up may be performed with minimal equipment, such as a plyometric box and added resistance to achieve PAP effects from AEL. ${ }^{7}$ Using resistance bands or adjustable dumbbells helps to tailor AEL to fit the particular needs of a given athlete, based on factors such as age, gender, training experience, and current training status. ${ }^{5}$

The data regarding various loading considerations are not yet clear due to a variety of protocols being implemented in the current research. TL is most often used as a preloading condition to CMJ execution as it occurs naturally and is easily performed without the need for an external load. However, results from recent studies ${ }^{3,5-7}$ reveal that AEL is in most cases superior to TL, or at the least equivalent. Educators should be aware that the implementation of AEL as a "one-size-fits-all" approach is not the best approach when aiming to obtain peak outcomes. Prior levels of relative strength must be considered as a necessary strength level must be achieved to experience acute benefits during explosive movements. ${ }^{2}$ In addition, the authors stated that the magnitude of the AEL resistance could have a vital impact on the outcomes based on prior neuromuscular and architectural adaptations from intense training. Until more attention is given to establishing clear guidelines for AEL loading considerations, no specific recommendations can be given.

A recent review on AEL for training and performance stated that more research involving trained subjects is necessary before credible conclusions can be drawn. ${ }^{2}$ Future research should focus on constructing more definite recommendations for loading magnitude and volume as it relates to the application of AEL for acute jump performance. As previously mentioned, prior levels of strength must be considered as this could impact the considerations for optimal loading. There is a noticeable shortage of studies involving female participants. Gathering more data on the female population would provide a greater breadth of understanding as each of the studies examined in this CAT was composed of exclusively male participants. The implications of AEL as a training method for chronic jump performance have been examined on a small scale and are beyond the scope of this critical appraisal. It is suggested that AEL research should focus initially on acute adaptations to allow for optimal execution before proceeding to AEL's impact on chronic adaptations. ${ }^{2}$ A possible direction for future studies would be to utilize AEL for both training sessions and warm-up in an attempt to benefit both acute and chronic jump performance. Research in this area would have a wider application for team sports such as volleyball, basketball, football, and soccer.

\section{Conclusion}

AEL appears to yield a positive effect on acute CMJ performance in adult, resistance-trained males compared with TL. While more research is needed to make a definitive recommendation on the topic, athletes are encouraged to employ AEL during warm-up 2 to 6 minutes before activities, in which optimal performance is classified as single action or short and explosive in nature. The ideal AEL loading ranges from $5 \%$ to $25 \% \mathrm{BM}$ and $105 / 125$ repetition maximum but must be determined beforehand as each athlete and scenario differs based on specific demands and physiological differences. This CAT should be reviewed in 2 years to determine whether additional best-research evidence has been published that could aid in answering the focused clinical question. 


\section{References}

1. Douglas J, Pearson S, Ross A, McGuigan M. Chronic adaptations to eccentric training: a systematic review. Sports Med. 2017;47(5): 917-941. PubMed ID: 27647157 doi:10.1007/s40279-016-0628-4

2. Wagle JP, Taber CB, Cunanan AJ, et al. Accentuated eccentric loading for training and performance: a review. Sports Med. 2017; 47(12):2473-2495. PubMed ID: 28681170 doi:10.1007/s40279-0170755-6

3. Majeedkutty NA, Yiing PS, Paul A. Accentuated eccentric training: effects on horizontal jump distance and muscle strength among young adults. MOJ Yoga Physical Ther. 2018;3(3):59-62. doi:10.15406/ mojypt.2018.03.00045

4. Sheppard J, Newton R, McGuigan M. The effect of accentuated eccentric load on jump kinetics in high-performance volleyball players. Int J Sports Sci Coach. 2007;2(3):267-273. doi:10.1260/ 174795407782233209

5. Ong JH, Lim J, Chong E, Tan F. The effects of eccentric conditioning stimuli on subsequent counter-movement jump performance. $J$ Strength Cond Res. 2016;30(3):747-754. PubMed ID: 26332775 doi:10.1519/ JSC.0000000000001154

6. Bridgeman LA, McGuigan MR, Gill ND, Dulson DK. The effects of accentuated eccentric loading on the drop jump exercise and the subsequent postactivation potentiation response. J Strength Cond Res. 2017;31(6):1620-1626. PubMed ID: 28538313 doi:10.1519/ JSC.0000000000001630
7. Hughes JD, Massiah RG, Clarke RD. The potentiating effect of an accentuated eccentric load on countermovement jump performance. $J$ Strength Cond Res. 2016;30(12):3450-3455. PubMed ID: 27100315 doi:10.1519/JSC.0000000000001455

8. Oxford Centre for Evidence-Based Medicine. Levels of evidence. 2011. https://www.cebm.ox.ac.uk/resources/levels-of-evidence/ocebmlevels-of-evidence. Accessed August 10, 2020.

9. Aboodarda SJ, Yusof A, Abu Osman NA, Thompson MW, Mokhtar $\mathrm{AH}$. Enhanced performance with elastic resistance during the eccentric phase of a countermovement jump. Int J Sports Physiol Perform. 2013;8(2):181-187. doi:10.1123/ijspp.8.2.181

10. Walshe AD, Wilson GJ, Ettema GJ. Stretch-shorten cycle compared with isometric preload: contributions to enhanced muscular performance. J Appl Physiol. 1998;84(1):97-106. PubMed ID: 9451623 doi:10.1152/jappl.1998.84.1.97

11. Cronin JB, McNair PJ, Marshall RN. Magnitude and decay of stretch-induced enhancement of power output. Eur J Appl Physiol. 2001;84(6):575-581. PubMed ID: 11482554 doi:10.1007/s0042101 00433

12. Frizziero A, Trainito S, Oliva F, Aldini NN, Masiero S, Maffulli N. The role of eccentric exercise in sport injuries rehabilitation. $\mathrm{Br} \mathrm{Med}$ Bull. 2014;110(1):1-29. doi:10.1093/bmb/ldu006

13. LaStayo P, Marcus R, Dibble L, Frajacomo F, Lindstedt S. Eccentric exercise in rehabilitation: safety, feasibility, and application. $J$ Appl Physiol. 2013;116(11):1426-1434. PubMed ID: 23823152 doi:10. 1152/japplphysiol.00008.2013 